\title{
Clinical experience with tixocortol pivalate
}

\author{
STEPHEN B. HANAUER, MD
}

\begin{abstract}
Tixocortol pivalate, the 21-thiol derivative of hydrocortisone, is a newly synthesized steroid with topical anti-inflammatory properties but is devoid of systemic glucocorticoid and mineralocorticoid activities and toxicity. The mechanism of actios appears to be similar to that of other corticosteroids with regard to steroid binding sites and prostaglandin biosynthesis, however, the disassociation between local and systemic effects is due to a 'first pass' liver metabolism and rapid transformation within red blood cells. In both open and controlled studies in patients with acute or recurrent distal colitis, clinical efficacy has been noted at doses of $250 \mathrm{mg}$ to $1000 \mathrm{mg}$ in $100 \mathrm{~mL}$ solution without an effect on serum cortisol levels or blood chemistries. Tixocortol pivalate has consistently shown to be as effective as systemically absorbed steroids for the treatment of distal ulcerative colitis without the undesirable effects associated with currently available steroid preparations. Can J Gastroenterol $1988 ; 2(4): 156-8$
\end{abstract}

Key Words: Anti-inflammatory, Steroid, Ulcerative colitis

U LCERATIVE COLITIS REMAINS A DISorder of unknown etiology (1). Despite potentially life threatening pancolonic and transmural inflammation, the inflammatory response is limited to the mucosa of the rectum and sigmoid in the majority of cases (2). The superficial nature and limited extent of the inflammation often make ulcerative colitis amenable to local (intrarectal) treatment.
Shortly after the introduction of oral cortisone as therapy for ulcerative colitis, Truelove $(3,4)$ described the use of topical hydrocortisone by rectal drip. Subsequent randomized, controlled trials by Truelove (5) and Watkinson (6) confirmed the effectiveness of $100 \mathrm{mg}$ intrarectal doses of hydrocortisone hemisuccinate solutions compared to placebo in achieving clinical, sigmoidoscopic and histologic improvement of active dis-

Department of Medicine, University of Chicago Medical Center, Chicago, Illinois

Correspondence and reprints: Dr Stephen B. Hanauer, Assistant Professor of Medicine, Section of Gastroenterology, University of Chicago, 5841 South Maryland Avenue, Chicago, IL 60637, USA

This paper was presented at Falk Symposium No $49^{\prime}$ 'Trends in inflammatory bowel disease therapy' held at Lake Louise, Alberta, April 20-23, 1988

ease. In the United States, $100 \mathrm{mg}$ hydrocortisone retention enemas (Cortenema; Reid-Rowell) have become the most widely prescribed local ther apy for distal colitis. However, continuous therapy with topical hydrocortisone is complicated by the consequences of significant systemic absorption proportional to the administered dose and chronicity of treatment (7-9).

Tixocortol pivalate (pregn-4-ene3,20-dione-21-thiol-11b, 17 dihydroxy21-pivalate), the 21-thiol derivative of hydrocortisone (Figure 1), is a newly synthesized steroid with anti-inflammatory

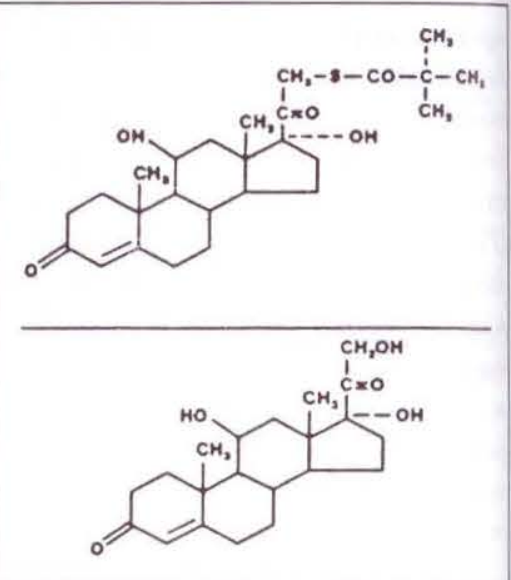

Figure 1) Molecular structures of tixocortol pivalate (top) and hydrocortisone 
properties in animal models of inflammation (Sandoz Pharmaceuticals, data on file). Moreover, it has the unique feature of topical anti-inflammatory effects without the typical hormonal and metabolic effects of systemic absorption (11). Hence, tixocortol pivalate is considered the first of a new class, the 'nonsystemic steroids.

The mechanism of action of tixocor tol pivalate appears to be similar to that of other corticosteroids with regard to steroid binding sites and prostaglandin biosynthesis. However, in both animal and human studies, tixocortol pivalate was devoid of systemic glucocorticoid and mineralocorticoid activities, as well as toxicity, in most cases at dose levels as high as $4000 \mathrm{mg} / \mathrm{kg}$. While tixocortol pivalate is absorbed to the same extent as other steroids, including hydrocortisone, the disassociation between local and systemic effects is due to a 'first-pass' liver metabolism and rapid transformation within red blood cells $(11,12)$.

\section{CLINICAL STUDIES}

Open studies: Both biological tolerance studies in patients with refractory proctitis and multicentre open studies in patients with acute or recurrent distal colitis have confirmed the absence of an effect of tixocor tol pivalate on serum cortisol levels or other blood chemistries, and apparent clinical efficacy using doses of $250 \mathrm{mg}$ in $100 \mathrm{~mL}$ solutions (Sandoz Pharmaceuticals, data on file).

Controlled studies: Two multicentre controlled studies performed outside of the United States compared $250 \mathrm{mg}$ tixocortol pivalate enemas to $5 \mathrm{mg}$ betamethasone phosphate with all patients receiving between 1.5 and $4 \mathrm{~g}$ sulfasalazine. Over the three week course of the studies, both enemas were well tolerated and equally effective in reducing the symptoms of ulcerative colitis, vet only the betamethasone increased serum cortisol (Sandoz Pharmaceuticals, data on file).

In the United States, three large, controlled, double-blind, randomized, multicentre studies have compared 250 $\mathrm{mg}$ tixocor tol pivalate enemas to $100 \mathrm{mg}$ hydrocortisone enemas (Cortenema; Reid-Powell) in a total of 337 patients with left sided ulcerative colitis who were not receiving oral or local steroids, although continued therapy with sulfasalazine was allowed and analyzed separately $(13,14)$. The trials all lasted for three weeks with weekly evaluations of symptoms and signs of ulcerative colitis, hematologic and clinical chemistry parameters, as well as determinations of 08:00 serum cortisol levels. Proctoscopic examinations were performed at baseline and after 14 and 22 days

Symptoms of diarrhea, rectal bleeding, abdominal pain, rectal pain and general malaise statistically improved in both treatment groups, as did the number of bowel movements and the number of bowel movements accompanied by blood. There was a direct correlation with the severity of the symptoms at baseline and the degree of improvement; worse initial symptoms were associated with a greater degree of improvement. Likewise, endoscopic findings of colonic hyperemia, ulceration and the physician's overall rating of the colitis improved in both treatment groups by week 2 and continued to improve, such that by the end of the three week study, $68 \%$ of the tixocor tol pivalate and $75 \%$ of the hydrocor tisone group had improved, and 34\% of the tixocortol patients and $36 \%$ of the hydrocortisone patients had healed (neither proportion was statistically significant between groups) (Figure 2). Patients treated with sulfasalzine also improved in both groups.

Adverse reactions were typically mild, if reported, and no significant steroid related side effects were observed in the tixocor tol treated patients. The only consistently significant laboratory parameters found to be altered were the white blood count which was increased (increased neutrophils, decreased lymphocytes and eosinophils) and the serum potassium which was decreased in the hydrocortisone treated patients. Serum cortisol determinations, when measured, were not reduced in either group at three weeks.

Hence, after three weeks, tixocortol pivalate was equally effective as hydrocortisone therapy without systemic glucocorticoid or mineralocorticoid effects.

Subsequently, a short term, four day study was performed comparing $250 \mathrm{mg}$ tixocortol pivalate to a saline placebo enema in 24 patients with ulcerative colitis or Crohn's disease of the rectum. During this brief study, the symptoms of diarrhea, rectal bleeding and number of bowel movements, as well as proctoscopic signs of erythema, granularity, mucopus and overall rating of colitis improved in the tixocortol patients, whereas no changes in symptoms or signs of inflammatory bowel disease were observed in the placebo group (Tables 1 and 2).

A randomized, double-blind, parallel group study also was conducted in 30 patients with ulcerative colitis to elucidate a dose response with higher doses of tixocor tol pivalate. Ten patients were allocated to receive either $250 \mathrm{mg}, 500$ $\mathrm{mg}$ or $1000 \mathrm{mg}$ tixocor tol pivalate in 100

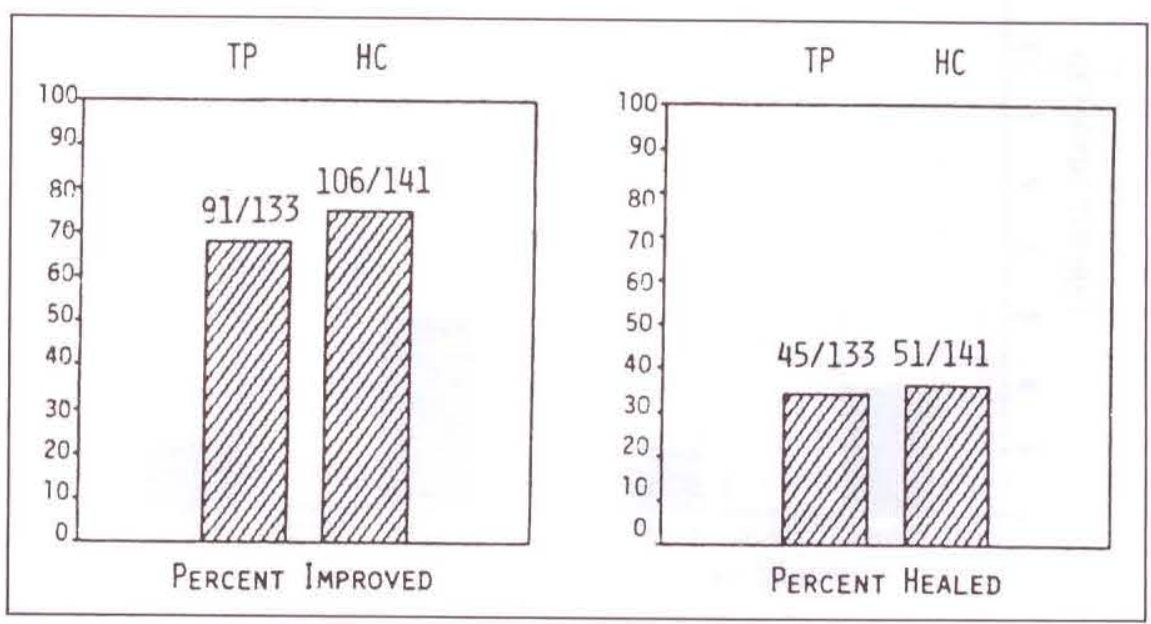

Figure 2) Summary of three US controlled trials of tixocortol pivalate (TP) versus hydrocortisone (HC) enemas in acute ulcerative colitis: Outcome after three weeks 
TABLE 1

\section{Physician rating of signs on day 4}

\begin{tabular}{lc}
\hline Mucosal vascular pattern & 0 \\
Erythema & $\mathrm{T}<\mathrm{P}$ \\
Granularity & $\mathrm{T}<\mathrm{P}$ \\
Friability & 0 \\
Ulcerations & 0 \\
Mucopus & $\mathrm{T}<\mathrm{P}$ \\
Summed severity index & $\mathrm{T}<\mathrm{P}$ \\
\hline
\end{tabular}

TTixocortol pivalate: P Placebo

$\mathrm{mL}$ solution over three weeks. At the conclusion of the study, no dose response was identified in the entire group (Figure 3) or after analysis of the subgroups of sulfasalazine treated or refractory patients.

\section{CONCLUSION}

Tixocortol pivalate, the first of a new class of nonsystemic steroids devoid of glucocorticoid and mineralocorticoid effects, has consistently been shown to be as effective as systemically absorbed steroids for the treatment of distal ulcerative colitis. Thus, it appears that therapy with tixocortol pivalate offers the advantage of steroid therapy without the undesirable effects previously associated with currently available steroid preparations. Future studies will focus on longer duration placebo controlled enema trials as well as the development of oral, targeted delivery systems for the potential use in proximal colitis and Crohn's disease.

TABLE 2

Patient rating of symptoms compared to baseline

\begin{tabular}{|c|c|c|c|c|c|}
\hline \multirow[b]{2}{*}{ Parameter } & \multicolumn{3}{|c|}{$\begin{array}{l}\text { Tixocortol pivalate } \\
\text { Day }\end{array}$} & \multicolumn{2}{|r|}{$\begin{array}{l}\text { Placebo } \\
\text { Day }\end{array}$} \\
\hline & 2 & 3 & 4 & 2 & 3 \\
\hline Diarrhea & 0 & 0 & $<$ & 0 & 0 \\
\hline Rectal bleeding & 0 & $\prec$ & $<$ & 0 & 0 \\
\hline Abdominal pain & 0 & 0 & 0 & 0 & 0 \\
\hline Rectal pain & - & - & - & - & - \\
\hline Nausea & - & - & - & - & - \\
\hline Vomiting & - & - & - & - & - \\
\hline Change in appetite & 0 & 0 & 0 & 0 & 0 \\
\hline Feeling of illness & 0 & $\prec$ & $<$ & 0 & 0 \\
\hline Number of bowel movements & 0 & $<$ & $<$ & 0 & 0 \\
\hline $\begin{array}{l}\text { Number of bowel movements } \\
\text { with blood }\end{array}$ & 0 & $\prec$ & $<$ & 0 & 0 \\
\hline
\end{tabular}

\section{REFERENCES}

1. Kirsner JB, Shorter RG. Recent developments in 'non-specific' inflammatory bowel disease. $\mathrm{N}$ Engl J Med 1982;306:775-85, 837-48.

2. Edwards FC, Truelove SC. The course and prognosis of ulcerative colitis. Part II. Long-term prognosis. Gut 1963:4:309-15

3. Truelove SC. Treatment of ulcerative colitis with local hydrocortisone. Br Med J 1956;2:1267-72.

4. Truelove SC. Treatment of ulcerative colitis with local hydrocor tisone hemisuccinate sodium. Br Med J $1957 ; 1: 1437-43$.

5. Truelove SC. Treatment of ulcerative colitis with local hydrocortisone hemisuccinate sodium. A report on a controlled therapeutic trial. Br Med J 1958;2:1072-7.

6. Watkinson G. Treatment of ulcerative colitis with topical hydrocortisone

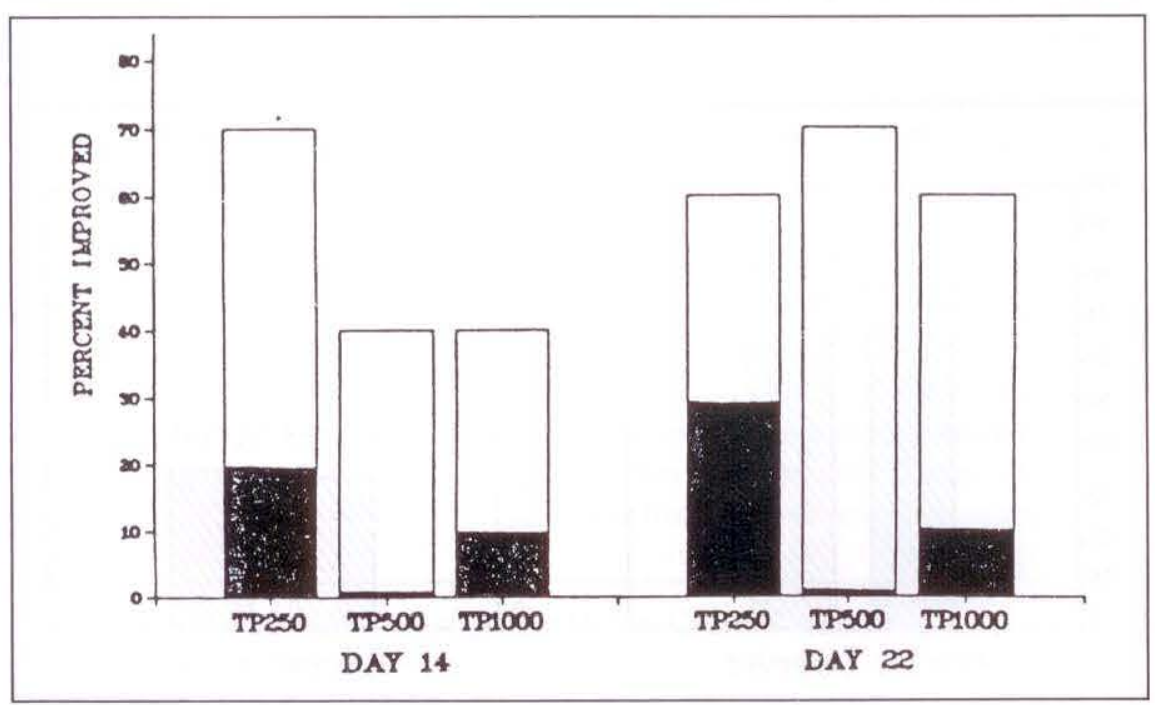

Figure 3) Percentage of patients healed doses of tixocortol pivalate (TP) hemisuccinate sodium. A controlled trial employing restricted sequential analysis. Br Med J 1958;2:1077-82.

7. Sherman LF, Tenner RJ, Schottler JL Treatment of ulcerative colitis with hydrocortisone alcohol retention enemas. A topical and systemic anti-inflammatory agent. Part II. Dis Colon Rectum 1967;10:43-52.

8. Goldstein MJ, Gelzayd EA, Kirsner JB Some observations on the hazards of corticosteroid therapy in patients with inflammatory bowel disease. Trans Am Acad Ophthalmol Otolaryngol 1967;71:254-61.

9. Singleton JW. Medical therapy of inflammatory bowel disease. Med Clin North Am 1980;64:1117-33.

10. Davies JE, Kellet DN, Staniforth MW, et al. Pharmacological study of a new anti-inflammatory steroid, tixocortol pivalate (TO 1016). Arzneimittelforsch 1981;31:453-9

11. Larochelle P, Du Souich P, Colte E, eta Tixocortol pivalate, a corticosteroid with no systemic glucocor toid effect after oral, intrarectal and intranasal application. Clin Pharmacol Ther 1983;33:343-50.

12. Goyer R, Leveille J, Larochelle P, et al. Preliminary report on the fate of tixocor tol pivalate (TP), a topical steroid without systemic effects. Presented at the World conference on Clinical Pharmacology Therapeutics, Washing ton, DC, August 1983.

13. Levinson RA. Intrarectal treatment of ulcerative colitis with tixocor tol pivalate, a topical, nonsytemic antiinflammatory steroid, comparison with hydrocortisone enema. Gastroenterology 1986;90:1520.

14. Hanauer SB, Kirsner JB, Barrett WE. The treatment of left-sided ulcerative colitis with tixocor tol pivalate (TP). Gastroenterology 1986;90:1449. 


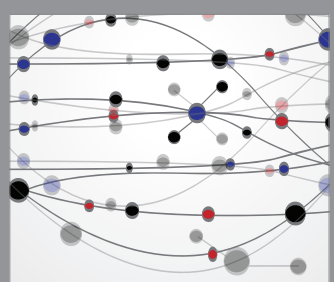

The Scientific World Journal
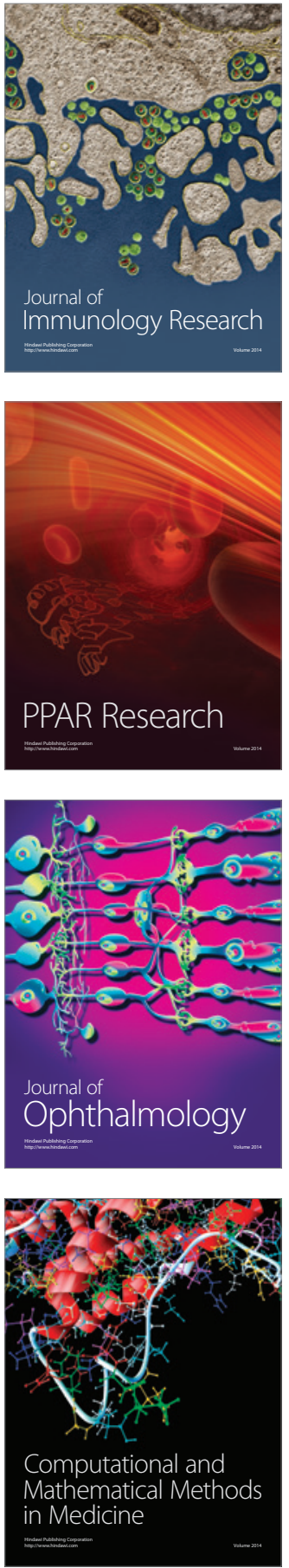

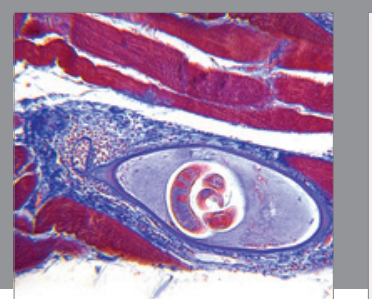

Gastroenterology Research and Practice

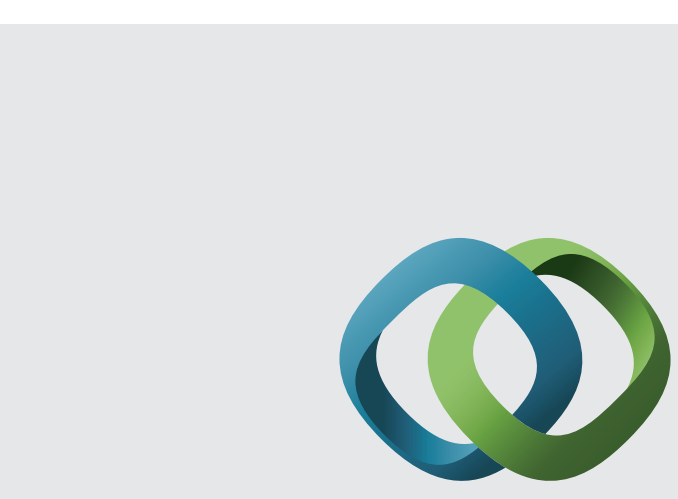

\section{Hindawi}

Submit your manuscripts at

http://www.hindawi.com
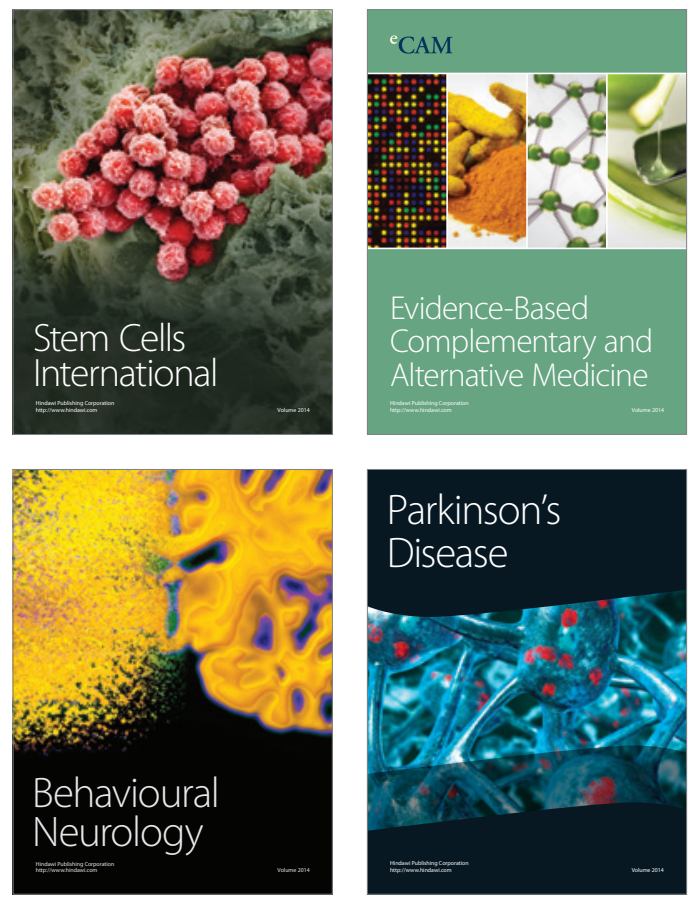
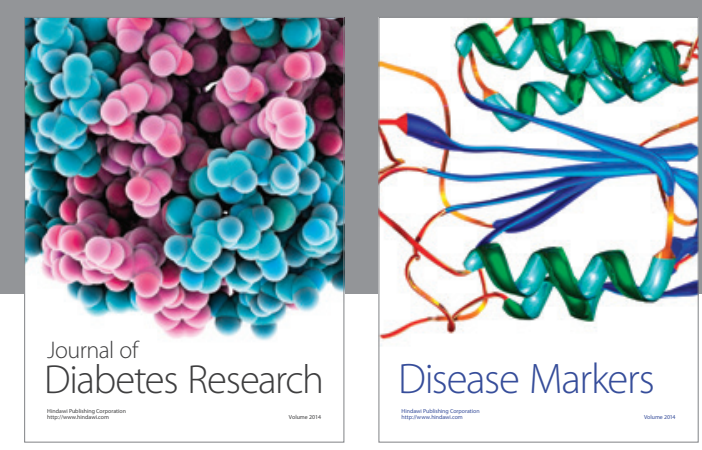

Disease Markers
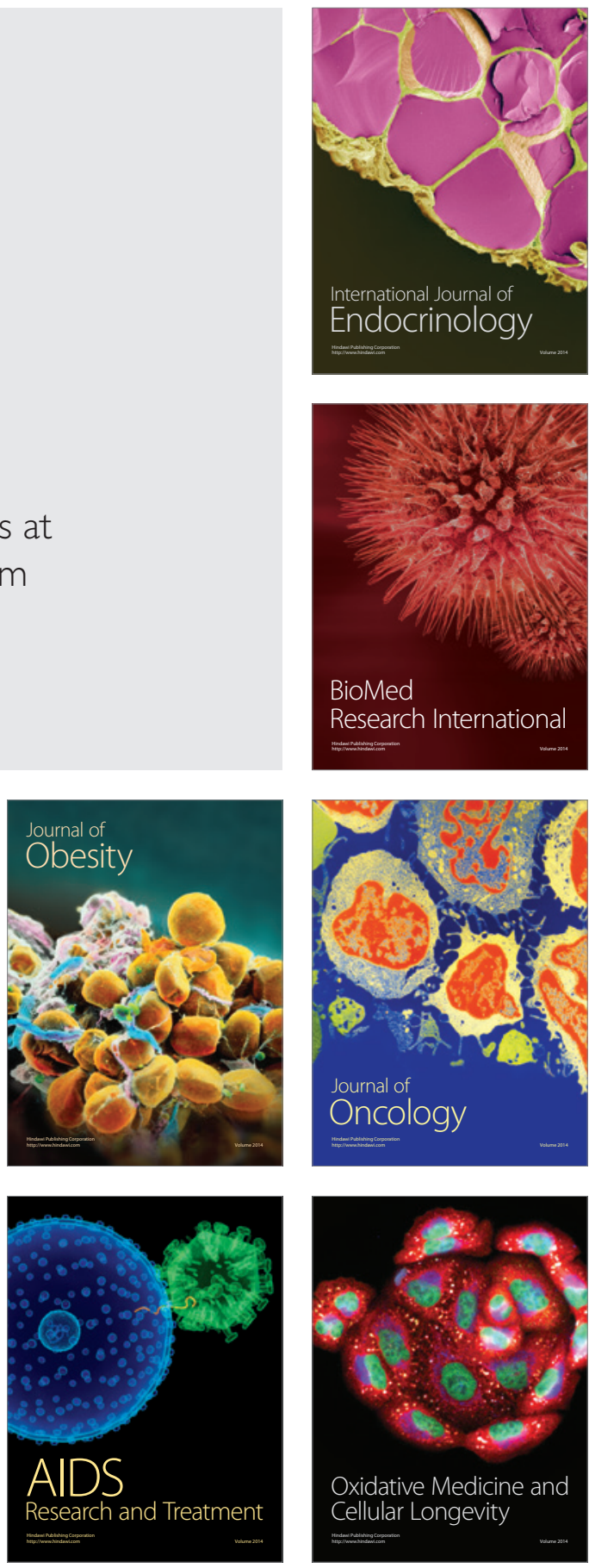